Doi: HTTPS://DOI.ORG/10.23910/IJBSM/2018.9.1.3C0908

\title{
Stability Analysis for Grain Yield and its Components in Soybean (Glycine max L. Merrill)
}

\author{
Ranjeet Singh Jakhar ${ }^{*}$, P. S. Salke ${ }^{2}$, A. M. Misal ${ }^{3}$, V. G. Sonawane ${ }^{4}$, K. Srikanth ${ }^{5}$, S. R. Borade ${ }^{6}$ and Rajveer ${ }^{7}$ \\ ${ }^{123456}$ Dept. of Agriculture Botany, College of Agriculture, Latur, VNMKV, Parbhani, M.S. (431 402), India \\ ${ }^{7}$ Dept. of Soil Science, SHIATS, Allahabad, U.P. (211 007), India
}

\section{Corresponding Author}

Ranjeet Singh Jakhar

e-mail: ranjeetjakhar91@gmail.com

\author{
Article History \\ Article ID: 3 C0908 \\ Received in $02^{\text {nd }}$ November, 2017 \\ Received in revised form $17^{\text {th }}$ December, 2017 \\ Accepted in final form $29^{\text {th }}$ January, 2018
}

\begin{abstract}
The present investigation was conducted with twenty two genotypes including five checks of soybean (Glycine max L.) for stability performance over three environments in a R.B.D. with three replication during kharif-2016. The analysis of variance revealed significant differences among the genotypes for all traits studied over all environments, indicating sufficient amount of variability present. G XE interaction is highly significant for all character studied except plant height, number of pods per plant and oil content. Environment (linear) was highly significant for all traits except number of seeds per pod and oil content. The predominance of linear component would help in predicting the performance of genotypes across environment. Considering the nature of stability, two genotypes MAUS-740, MAUS-710 were found promising and they had stable performance over three environments for grain yield per plant ${ }^{-1}$ while MAUS-614 was suitable for favorable environment. For number of branches plant ${ }^{-1}$ two genotypes, KDS-980 and MACS-1460 showed superiority for average response and stability under all environments. For number of pod per plant three genotypes, KDS-1045, AMS-100-39-1 and MAUS-706 had stable performance in all three environments. For number of seeds pod ${ }^{-1}$ genotypes, AMS-1002, AMS-1003, MAUS-706 and MAUS-158 suitable under unfavorable environment.The genotypes, MAUS-740, MAUS-614 had stable performance over three environments for 100 seed weight. Use of genotype with wide (MAUS-740, MAUS-710) or specific stability (KDS-921, MACS-1543, AMS-MB-5-19, KDS-1045, AMS-100-39-1, MACS-1460, JS-9305, MAUS-706)) in development of new varieties with desired nature of adaptability suggested.
\end{abstract}

Keywords: Stability analysis, soybean genotypes, yield plant ${ }^{-1}$

\section{Introduction}

Soybean (Glycine max (L.) Merill) is also known as 'golden bean' and miracle crop which is an efficient producer of two most scarce items in the world food economy i.e. high quality protein (40\%) and oil (20\%). In India, the area under soybean during kharif- 2015-16 was 109.71 lakh ha with total production of 114.90 lakh MT with an average productivity of $1047 \mathrm{~kg} \mathrm{ha}^{-1}$. India ranks fifth in area and production of soybean in the world after USA, Brazil, China and Argentina. Major soybean growing states in India are Madhya Pradesh, Maharashtra, Rajasthan, Andhra Pradesh and Karnataka etc. Madhya Pradesh ranking $1^{\text {st }}$ in production of soybean. Soybean was introduced in Maharashtra state during the year 1984-85 and it was grown only on 5.6 lakh ha until 1994 but now a day's area under soybean is increasing largely. In Maharashtra area under soybean during kharif - 2016 was 35.80 lakh ha with total production 39.45 lakh MT with an average productivity of $1102 \mathrm{~kg} \mathrm{ha}^{-1}$. For advancement or breakthrough in production in any agricultural crop, the prime requirement is the availability of appropriate genotype. In order to identify superior genotypes that can give reproducible performance (Stable) breeders evaluate the breeding materials over a number of locations or seasons or both. The differential response of genotypes with the varying environments i.e. genotypes environment (GE) interaction, creates the problem in the selection of ideal genotypes over a wide range of environments. A genotype with smallest $\mathrm{s} 2 \mathrm{gl}$ is regarded as most stable genotype. Statistical approach of Finely and Wilkinson (1963) proved considerably useful to measure the phenotypic stability in the performance of genotype. He considered the linear regression sole (bi) as measure of stability. This regression analysis proposed by Finely and Wilkinson (1963) was improved by Eberhart and Russel (1966) by introduction of one more parameter, $\left(S^{2} \mathrm{di}\right)$ which accounts for unpredictable irregularities in response of genotypes to varying environments. Later on Paroda and Hays (1971) stressed that linear regression of variety be considered for evaluating the potential, whereas deviation around regression gives a measure of stability of genotype over environments. Considering all the above points, present investigation was undertaken in soybean with an object to estimate stability parameters for grain yield and its important components. 


\section{Materials and Methods}

The experimental materials comprised of 17 promising newly developed genotypes of soybean developed at different centres of Maharashtra and five checks viz., JS 335, JS 9305, JS-97-52, MAUS 71, and MAUS 158 were used. These genotypes were sown on three different sowing dates during kharif 2016, which created three environments as E1 (Latur), E2 (Parbhani), E3 (Badnapur), respectively. The experiment was laid in randomized block design with three replications maintaining $45 \times 5 \mathrm{~cm}^{2}$ spacing between rows and plants, respectively. Observations were recorded on 11 characters viz., number of days to $50 \%$ flowering, days to maturity, plant height, number of branches per plant, number of pods per plants, seeds pods ${ }^{-1}, 100$ seed weight, seed yield plot ${ }^{-1}$, oil content, protein content (\%), and seed yield per plant. Stability analysis was done as per the procedure suggested by Eberhart and Russel (1966).

\section{Results and Discussion}

The analysis of variance representing the mean sum of square due to different sources of variation as per Eberthart and Russel (1966) stability analysis is presented in Table 1. Pooled analysis of variance over three different environments showed genotypic variances, when tested against pooled error were

\begin{tabular}{|c|c|c|c|c|c|c|c|c|c|}
\hline Sr. No. & Character & Genotype & Environment & GXE & Env $+(\mathrm{G} \times \mathrm{E})$ & Env (L) & $\mathrm{G} \times \mathrm{E}(\mathrm{L})$ & PD & $\mathrm{PE}$ \\
\hline & DF & 21 & 2 & 42 & 44 & 1 & 21 & 22 & 126 \\
\hline 1. & Days to $50 \%$ flowering & $12.98^{* *++}$ & $2.07^{* *}$ & $1.26^{* *}$ & $1.30^{* *}$ & $4.14^{* *}$ & $0.87^{* *}$ & $1.57^{* *+}$ & 0.10 \\
\hline 2. & Days to maturity & $50.53^{* *++}$ & $23.16^{* *++}$ & $2.30^{* *++}$ & $3.27^{* *++}$ & $46.33^{* *++}$ & $3.78^{* *++}$ & $0.83^{* *++}$ & 0.06 \\
\hline 3. & Plant height & $168.3^{* *_{++}}$ & $191.87^{* *_{++}}$ & $5.30^{+}$ & $13.78^{* *_{++}}$ & $383.74^{* *++}$ & $7.73^{++}$ & 2.74 & 6.16 \\
\hline 4. & No. of branches plant ${ }^{-1}$ & $0.14^{* *++}$ & 0.021 & $0.015^{* *}$ & $0.015^{* *}$ & $0.041^{*}$ & $0.019^{* *}$ & 0.011 & 0.007 \\
\hline 5. & No. of pod plant ${ }^{-1}$ & $81.27^{* *++}$ & $71.36^{* *++}$ & $2.68^{++}$ & $5.80^{++}$ & $142.72^{* *++}$ & $4.87^{++}$ & 0.47 & 5.69 \\
\hline 6. & No. of seeds pod ${ }^{-1}$ & $0.078^{* *++}$ & 0.005 & $0.012^{* *}$ & $0.011^{*}$ & 0.010 & $0.014^{* *}$ & 0.009 & 0.006 \\
\hline 7. & 100 seed weight & $3.36^{* *++}$ & $24.35^{* *++}$ & $0.21^{* *_{+}}$ & $1.30^{* *++}$ & $48.71^{* *++}$ & $0.325^{* *++}$ & $0.09^{*+}$ & 0.05 \\
\hline 8. & Grain yield plant ${ }^{-1}$ & $23.99^{* *++}$ & $98.96^{* *++}$ & $1.06^{*++}$ & $5.50^{* *++}$ & $197.91^{* *++}$ & $1.83^{* *++}$ & $0.27^{* *}$ & 0.69 \\
\hline 9. & Grain yield plot ${ }^{-1}$ & $0.033^{* *}$ & $1.108^{* *++}$ & $0.031^{* *}$ & $0.080^{* *++}$ & $2.21^{* *++}$ & $037^{* *}$ & $0.023^{* *++}$ & 0.010 \\
\hline 10. & Oil content & $0.698^{* *++}$ & 0.054 & 0.023 & 0.025 & $0.107^{+}$ & 0.027 & 0.019 & 0.035 \\
\hline 11. & Protein content & $6.54^{* *++}$ & 0.033 & $0.090^{* *}$ & $0.087^{* *}$ & $0.066^{*}$ & $0.077^{* *}$ & $0.098^{++}$ & 0.014 \\
\hline
\end{tabular}

PD: Pooled deviation; PE: Pooled error; * and ** indicates significant at $(p=0.05)$ and $(p=0.01)$, against pooled error respectively; + and ++ indicates significant at $(p=0.05)$ and $(p=0.01)$, against pooled deviation respectively

highly significant for all traits and when tested against pooled deviation it is highly significant for all traits except grain yield per plot. The difference due to genotypes and environments indicates presence of variation among genotypes as well as among environments. While for environment variance it shows highly significant for traits viz. days to $50 \%$ flowering, days to maturity, plant height, number of pods plant ${ }^{-1}, 100$ seed weight, yield plant ${ }^{-1}$ and yield plot $^{-1}$ when tested against pooled error but when tested against pooled deviation it showed significant for days to maturity, plant height, number of pods plant ${ }^{-1}, 100$ seed weight, yield plant ${ }^{-1}$ and yield plot 1. For GXE interaction, when tested against pooled error it showed significant for all data except plant height, number of pods plant ${ }^{-1}$, and protein content but when tested against pooled deviation it shows significant for traits viz. days to maturity, plant height, number of pods plant ${ }^{-1}, 100$ seed weight and yield plant ${ }^{-1}$. Mean square due to pooled deviation was found significant for traits viz. days to $50 \%$ flowering, days to maturity, 100 seed weight, yield plant ${ }^{-1}$ and yield plot $^{-1}$ when tested against pooler error.

Environment indices for 11 characters given in Table 2 showed
Table 2: Estimates of environmental indices for each character under different environment

\begin{tabular}{|c|c|c|c|}
\hline \multirow[t]{2}{*}{ Observations } & \multicolumn{3}{|c|}{ Environments } \\
\hline & $\begin{array}{c}\mathrm{E}_{1} \\
\text { (Latur) }\end{array}$ & $\begin{array}{c}E_{2} \\
\text { (Parbhani) }\end{array}$ & $\begin{array}{c}\mathrm{E}_{3} \\
\text { (Badnapur) }\end{array}$ \\
\hline Days to $50 \%$ flowering & -0.348 & 0.121 & 0.227 \\
\hline Days to maturity & -0.975 & 1.071 & $-0 . .96$ \\
\hline Plant height $(\mathrm{cm})$ & 3.236 & -2.548 & -0.688 \\
\hline No. of branches plant ${ }^{-1}$ & -0.019 & 0.035 & -0.016 \\
\hline No. of pods plant ${ }^{-1}$ & 2.032 & -1.398 & -0.634 \\
\hline Number of seeds pod ${ }^{-1}$ & 0.002 & 0.014 & -0.016 \\
\hline 100 seed weight $(\mathrm{g})$ & 1.174 & 0.857 & -0.317 \\
\hline Grain yield plant $^{-1}(\mathrm{~g})$ & 2.410 & -1.580 & -0.830 \\
\hline Grain yield plot $^{-1}(\mathrm{~kg})$ & 0.014 & 0.217 & -0.231 \\
\hline Oil content (\%) & -0.019 & 0.056 & -0.038 \\
\hline Protein content (\%) & 0.023 & -0.046 & 0.023 \\
\hline
\end{tabular}


that that $E_{1}$ environment was favourable for characters like plant height, number of pods per plant, 100 seed weight, grain yield per plant and protein content. In $\mathrm{E}_{2}$ environment was favourable for characters like days to maturity, number of branches, seed per pod, grain yield per plot and oil content. While $\mathrm{E}_{3}$ environment was favourable for days to $50 \%$ flowering and protein content.

\section{Stability Parameters of Genotypes}

On the basis of results of stability parameters (Table 3 ), the nature of stability of 22 genotypes for different characters has been discussed below.

\begin{tabular}{|c|c|c|c|c|c|c|c|c|c|c|}
\hline \multirow[t]{2}{*}{ SI. No. } & \multirow[t]{2}{*}{ Variety } & \multicolumn{3}{|c|}{ Days to $50 \%$ flowering } & \multicolumn{3}{|c|}{ Days to maturity } & \multicolumn{3}{|c|}{ Plant height } \\
\hline & & $\bar{X}$ & bi & $S^{2} d_{i}$ & $\bar{X}$ & bi & $S^{2} d_{i}$ & $\bar{X}$ & bi & $S^{2} d_{i}$ \\
\hline 1. & MACS-1460 & 39.33 & -0.186 & 0.111 & 95.00 & 1.849 & $0.958^{* *}$ & 51.33 & 1.977 & -4.456 \\
\hline 2. & KDS-904 & 43.56 & 0.245 & $0.847^{* *}$ & 97.89 & 1.032 & $0.879^{* *}$ & 49.60 & 2.317 & -4.759 \\
\hline 3. & MAUS-740 & 40.56 & 0.810 & 0.068 & 95.67 & 1.017 & $0.426^{* *}$ & 45.87 & $1.589^{*}$ & -6.101 \\
\hline 4. & AMS-1002 & 44.22 & -0.696 & $10.767^{* *}$ & 99.44 & 0.354 & $0.192^{*}$ & 54.09 & 1.487 & -4.739 \\
\hline 5. & KS-133 & 47.56 & 3.311 & 0.132 & 102.11 & 5.950 & $2.135^{* *}$ & 73.84 & 1.411 & -5.422 \\
\hline 6. & MACS-1543 & 42.22 & 0.702 & $4.766^{* *}$ & 100.67 & -0.554 & $1.291^{* *}$ & 54.69 & 1.043 & 7.088 \\
\hline 7. & KD-921 & 47.33 & 6.216 & $1.306^{* *}$ & 109.89 & 1.126 & 0.009 & 69.65 & 0.374 & -5.778 \\
\hline 8. & MAUS-614 & 41.56 & 0.810 & 0.068 & 95.56 & 1.495 & $0.193^{*}$ & 56.20 & 1.483 & -5.501 \\
\hline 9. & AMS-1003 & 42.00 & 0.568 & $1.835^{* *}$ & 95.33 & 0.557 & $2.841^{* *}$ & 49.82 & $0.374^{*}$ & -6.084 \\
\hline 10. & MACS-1505 & 41.22 & 1.832 & 0.228 & 91.00 & 0.278 & $0.663^{* *}$ & 53.60 & 1.405 & -6.058 \\
\hline 11. & AMS-100-39-1 & 41.33 & 4.279 & $1.123^{* *}$ & 92.22 & 0.572 & $1.990^{* *}$ & 55.49 & 0.696 & -4.357 \\
\hline 12. & MACS-1520 & 42.11 & 0.218 & -0.039 & 97.00 & 0.971 & -0.049 & 47.60 & 0.351 & -4.458 \\
\hline 13. & KDS-980 & 44.11 & -0.051 & $1.969^{* *}$ & 101.22 & 0.663 & -0.024 & 53.16 & 1.252 & 10.868 \\
\hline 14. & MAUS-706 & 41.00 & $0.003^{*}$ & -0.104 & 98.89 & 1.356 & $0.806^{* *}$ & 42.02 & 0.815 & -5.088 \\
\hline 15. & AMS-MB-5-19 & 43.11 & 1.052 & 0.206 & 99.78 & 0.263 & $1.199^{* *}$ & 53.60 & 0.720 & 5.337 \\
\hline 16. & KDS-1045 & 40.11 & 0.514 & $7.920^{* *}$ & 97.78 & -0.756 & $1.029^{* *}$ & 54.96 & 1.348 & -5.591 \\
\hline 17. & MAUS-710 & 41.33 & 1.213 & 0.285 & 95.44 & 1.234 & $1.469^{* *}$ & 45.00 & 1.100 & -5.970 \\
\hline 18. & JS-335(C) & 40.33 & 1.213 & 0.286 & 94.22 & 0.524 & $0.323^{*}$ & 45.27 & 0.463 & -5.294 \\
\hline 19. & JS-93-05(C) & 40.89 & 1.455 & 0.016 & 91.89 & 1.126 & 0.009 & 47.13 & 0.739 & -0.797 \\
\hline 20. & JS-97-52(C) & 43.11 & 2.450 & 0.175 & 97.67 & 2.636 & $0.195^{*}$ & 53.71 & -0.828 & -5.290 \\
\hline 21. & MAUS-71(C) & 42.00 & -5.564 & 0.081 & 94.33 & 0.509 & 0.059 & 53.33 & 1.221 & -5.899 \\
\hline \multirow[t]{4}{*}{22.} & MAUS-158(C) & 42.00 & 1.670 & 0.260 & 97.44 & -0.200 & $0.372^{* *}$ & 45.04 & 0.665 & -5.513 \\
\hline & Grand mean & 42.31 & & & 97.29 & & & 52.50 & & \\
\hline & SEm \pm & 0.89 & & & 0.64 & & & 1.171 & & \\
\hline & $\mathrm{SEb} \pm$ & 2.89 & & & 0.62 & & & 0.39 & & \\
\hline
\end{tabular}

Table 3 : Continue..

\begin{tabular}{|c|c|c|c|c|c|c|c|c|c|c|}
\hline \multirow[t]{2}{*}{ SI. No. } & \multirow[t]{2}{*}{ Variety } & \multicolumn{3}{|c|}{ No. of branches plant ${ }^{-1}$} & \multicolumn{3}{|c|}{ Number of pods plant ${ }^{-1}$} & \multicolumn{3}{|c|}{ Seeds pod ${ }^{-1}$} \\
\hline & & $\bar{x}$ & bi & $\mathrm{S}^{2} \mathrm{~d}_{\mathrm{i}}$ & $\bar{x}$ & bi & $S^{2} d_{i}$ & $\bar{x}$ & bi & $\mathrm{S}^{2} \mathrm{~d}_{\mathrm{i}}$ \\
\hline 1. & MACS-1460 & 3.02 & -0.789 & 0.012 & 48.64 & 1.514 & -5.356 & 2.89 & -4.917 & 0.003 \\
\hline 2. & KDS-904 & 2.96 & -0.789 & 0.012 & 38.33 & 0.787 & -5.411 & 2.93 & 8.688 & -0.005 \\
\hline 3. & MAUS-740 & 3.00 & 2.041 & 0.011 & 46.82 & 1.360 & -5.464 & 3.02 & -2.312 & -0.005 \\
\hline 4. & AMS-1002 & 2.78 & 0.680 & -0.005 & 39.53 & -0.533 & -4.814 & 2.93 & $0.004^{*}$ & -0.006 \\
\hline 5. & KS-133 & 2.67 & -5.805 & 0.009 & 49.09 & 2.469 & -5.184 & 2.60 & 14.767 & 0.007 \\
\hline 6. & MACS-1543 & 2.82 & 1.576 & $0.071^{* *}$ & 45.85 & 1.620 & -5.394 & 3.02 & 2.899 & 0.010 \\
\hline
\end{tabular}




\begin{tabular}{|c|c|c|c|c|c|c|c|c|c|c|}
\hline \multirow[t]{2}{*}{ SI. No. } & \multirow[t]{2}{*}{ Variety } & \multicolumn{3}{|c|}{ No. of branches plant ${ }^{-1}$} & \multicolumn{3}{|c|}{ Number of pods plant ${ }^{-1}$} & \multicolumn{3}{|c|}{ Seeds pod ${ }^{-1}$} \\
\hline & & $\overline{\mathrm{X}}$ & bi & $\mathrm{S}^{2} \mathrm{~d}_{\mathrm{i}}$ & $\overline{\mathrm{X}}$ & $\mathrm{bi}$ & $S^{2} d_{i}$ & $\overline{\mathrm{X}}$ & bi & $\mathrm{S}^{2} \mathrm{~d}_{\mathrm{i}}$ \\
\hline 7. & KD-921 & 2.18 & 4.442 & -0.005 & 39.80 & -0.517 & -3.454 & 2.40 & 3.477 & $0.023^{*}$ \\
\hline 8. & MAUS-614 & 3.04 & 0.680 & -0.005 & 51.29 & 1.357 & -5.138 & 3.16 & 6.373 & -0.004 \\
\hline 9. & AMS-1003 & 2.82 & 1.146 & 0.002 & 42.04 & -0.377 & -5.058 & 2.98 & 0.583 & 0.005 \\
\hline 10. & MACS-1505 & 3.07 & 1.934 & -0.005 & 44.58 & 0.371 & -4.982 & 2.96 & -4.917 & 0.003 \\
\hline 11. & AMS-100-39-1 & 2.93 & 2.041 & 0.011 & 49.20 & 0.858 & -5.451 & 2.98 & 2.320 & -0.005 \\
\hline 12. & MACS-1520 & 2.89 & 5.123 & -0.003 & 42.18 & -0.197 & -4.742 & 2.98 & -3.759 & 0.007 \\
\hline 13. & KDS-980 & 2.91 & $-1.255^{*}$ & -0.007 & 44.47 & 1.151 & -5.439 & 2.98 & 4.057 & -0.002 \\
\hline 14. & MAUS-706 & 2.67 & -1.936 & -0.005 & 46.33 & 1.265 & -5.196 & 3.00 & $0.004^{*}$ & -0.006 \\
\hline 15. & AMS-MB-5-19 & 2.71 & 9.924 & 0.006 & 59.20 & 1.853 & -5.237 & 3.00 & -1.733 & 0.001 \\
\hline 16. & KDS-1045 & 2.89 & $-2.509^{*}$ & -0.007 & 47.87 & 0.983 & -4.190 & 2.69 & -8.391 & -0.006 \\
\hline 17. & MAUS-710 & 2.89 & 3.080 & -0.004 & 54.38 & 2.509 & -5.361 & 2.96 & -2.312 & -0.005 \\
\hline 18. & JS-335(C) & 3.00 & 1.826 & -0.004 & 44.78 & 1.652 & -4.958 & 2.89 & -0.575 & 0.005 \\
\hline 19. & JS-93-05(C) & 2.91 & -1.363 & 0.001 & 37.53 & 0.768 & -5.267 & 2.93 & 6.951 & -0.002 \\
\hline 20. & JS-97-52(C) & 2.47 & $-0.001^{*}$ & -0.007 & 44.18 & 0.414 & -5.120 & 2.91 & -5.496 & $0.035^{*}$ \\
\hline 21. & MAUS-71(C) & 2.64 & -1.147 & 0.002 & 44.82 & 1.572 & -5.327 & 2.93 & 6.083 & 0.003 \\
\hline \multirow[t]{4}{*}{22.} & MAUS-158(C) & 3.09 & 3.080 & -0.004 & 41.78 & 1.123 & -5.441 & 3.02 & 0.293 & -0.003 \\
\hline & Grand mean & 2.83 & & & 45.58 & & & 2.92 & & \\
\hline & SEm \pm & 0.07 & & & 0.48 & & & 0.07 & & \\
\hline & SEb \pm & 2.39 & & & 0.27 & & & 4.39 & & \\
\hline
\end{tabular}

\begin{tabular}{|c|c|c|c|c|c|c|c|c|c|c|}
\hline \multirow[t]{2}{*}{ SI. No. } & \multirow[t]{2}{*}{ Variety } & \multicolumn{3}{|c|}{100 seed weight } & \multicolumn{3}{|c|}{ Yield plant $^{-1}$} & \multicolumn{3}{|c|}{ Yield plot ${ }^{-1}$} \\
\hline & & $\bar{x}$ & bi & $S^{2} d_{i}$ & $\bar{x}$ & $\mathrm{bi}$ & $S^{2} d_{i}$ & $\bar{x}$ & $\mathrm{bi}$ & $\mathrm{S}^{2} \mathrm{~d}_{\mathrm{i}}$ \\
\hline 1. & MACS-1460 & 12.52 & 0.550 & -0.035 & 17.15 & 0.673 & -0.561 & 1.66 & 1.721 & -0.006 \\
\hline 2. & KDS-904 & 14.73 & $1.604^{*}$ & -0.051 & 16.12 & 1.267 & -0.040 & 1.64 & 1.032 & -0.007 \\
\hline 3. & MAUS-740 & 13.34 & 1.066 & -0.046 & 17.97 & 1.113 & -0.667 & 1.64 & 0.198 & 0.025 \\
\hline 4. & AMS-1002 & 12.05 & 0.834 & 0.025 & 13.54 & 0.268 & -0.571 & 1.42 & 1.442 & -0.003 \\
\hline 5. & KS-133 & 13.25 & 0.820 & -0.008 & 16.48 & 0.939 & 0.018 & 1.58 & 1.829 & 0.004 \\
\hline 6. & MACS-1543 & 12.84 & 1.579 & -0.044 & 17.12 & $1.534^{*}$ & -0.672 & 1.69 & 1.417 & 0.005 \\
\hline 7. & KD-921 & 11.77 & 0.619 & -0.034 & 10.66 & -0.101 & -0.382 & 1.39 & 1.633 & -0.009 \\
\hline 8. & MAUS-614 & 14.96 & 0.996 & 0.017 & 23.19 & 1.375 & -0.648 & 1.86 & 0.931 & 0.008 \\
\hline 9. & AMS-1003 & 11.94 & 1.023 & 0.082 & 14.27 & 0.658 & -0.497 & 1.57 & 0.439 & -0.002 \\
\hline 10. & MACS-1505 & 12.77 & 0.574 & 0.059 & 16.16 & 0.278 & -0.599 & 1.62 & 0.665 & $0.045^{*}$ \\
\hline 11. & AMS-100-39-1 & 14.65 & 1.855 & 0.018 & 21.13 & $1.638^{*}$ & -0.673 & 1.75 & 0.807 & -0.009 \\
\hline 12. & MACS-1520 & 13.05 & 0.909 & -0.008 & 16.26 & 0.554 & -0.603 & 1.58 & 2.108 & 0.002 \\
\hline 13. & KDS-980 & 15.29 & 1.534 & -0.030 & 19.79 & 1.325 & -0.631 & 1.76 & 1.123 & -0.008 \\
\hline 14. & MAUS-706 & 13.79 & 1.323 & 0.009 & 18.83 & 1.402 & -0.062 & 1.57 & 0.708 & 0.004 \\
\hline 15. & AMS-MB-5-19 & 11.62 & 0.809 & -0.051 & 20.29 & 1.332 & -0.659 & 1.68 & 0.177 & -0.003 \\
\hline 16. & KDS-1045 & 11.98 & 1.330 & -0.048 & 14.96 & 1.441 & 0.840 & 1.68 & 0.572 & -0.001 \\
\hline 17. & MAUS-710 & 13.39 & 0.626 & 0.006 & 20.97 & 1.239 & 0.240 & 1.64 & 1.329 & $0.085^{* *}$ \\
\hline 18. & JS-335 (C) & 12.77 & 0.854 & $0.398^{* *}$ & 16.07 & 1.058 & -0.657 & 1.68 & 0.048 & $0.043^{*}$ \\
\hline
\end{tabular}




\begin{tabular}{|c|c|c|c|c|c|c|c|c|c|c|}
\hline \multirow[t]{2}{*}{ SI. No. } & \multirow[t]{2}{*}{ Variety } & \multicolumn{3}{|c|}{100 seed weight } & \multicolumn{3}{|c|}{ Yield plant $^{-1}$} & \multicolumn{3}{|c|}{ Yield plot ${ }^{-1}$} \\
\hline & & $\bar{x}$ & bi & $S^{2} d_{i}$ & $\bar{x}$ & $\mathrm{bi}$ & $S^{2} d_{i}$ & $\bar{X}$ & $\mathrm{bi}$ & $\mathrm{S}^{2} \mathrm{~d}_{\mathrm{i}}$ \\
\hline 19. & JS-93-05 (C) & 13.95 & 0.844 & 0.002 & 14.95 & 0.995 & -0.535 & 1.53 & $0.110^{*}$ & -0.010 \\
\hline 20. & JS-97-52 (C) & 12.32 & 0.703 & $0.155^{*}$ & 15.50 & 0.934 & -0.601 & 1.59 & 0.729 & $0.036^{*}$ \\
\hline 21. & MAUS-71 (C) & 13.17 & 0.515 & 0.110 & 16.70 & $0.923^{*}$ & -0.673 & 1.52 & 1.628 & $0.104^{* *}$ \\
\hline \multirow[t]{4}{*}{22.} & MAUS-158 (C) & 13.28 & 1.034 & $0.311^{* *}$ & 16.39 & 1.155 & -0.230 & 1.58 & $1.353^{*}$ & -0.010 \\
\hline & Grand mean & 13.16 & & & 17.02 & & & 1.62 & & \\
\hline & SEm \pm & 0.21 & & & 0.37 & & & 0.11 & & \\
\hline & SEb \pm & 0.20 & & & 0.17 & & & 0.48 & & \\
\hline
\end{tabular}

Table 3 : Continue..

\begin{tabular}{|c|c|c|c|c|c|c|c|}
\hline \multirow[t]{2}{*}{ SI. No. } & \multirow[t]{2}{*}{ Variety } & \multicolumn{3}{|c|}{ Oil content } & \multicolumn{3}{|c|}{ Protein content } \\
\hline & & $\bar{X}$ & $\mathrm{bi}$ & $\mathrm{S}^{2} \mathrm{~d}_{\mathrm{i}}$ & $\overline{\mathrm{X}}$ & $\mathrm{bi}$ & $\mathrm{S}^{2} \mathrm{~d}_{\mathrm{i}}$ \\
\hline 1. & MACS-1460 & 21.02 & -2.723 & -0.012 & 36.85 & -10.299 & $0.046^{*}$ \\
\hline 2. & KDS-904 & 20.24 & 2.587 & -0.026 & 38.51 & 3.701 & $0.337^{* *}$ \\
\hline 3. & MAUS-740 & 20.94 & 1.709 & -0.032 & 37.91 & 6.223 & 0.035 \\
\hline 4. & AMS-1002 & 20.44 & 3.537 & -0.012 & 35.97 & 3.464 & -0.011 \\
\hline 5. & KS-133 & 20.15 & -2.537 & -0.003 & 37.71 & -6.770 & $0.157^{* *}$ \\
\hline 6. & MACS-1543 & 20.12 & -1.996 & -0.003 & 39.46 & -6.076 & $0.114^{* *}$ \\
\hline 7. & KD-921 & 19.68 & 0.030 & 0.035 & 35.76 & -2.683 & $0.204^{* *}$ \\
\hline 8. & MAUS-614 & 20.15 & 2.371 & -0.034 & 34.46 & 0.777 & -0.013 \\
\hline 9. & AMS-1003 & 19.76 & 0.931 & 0.013 & 36.05 & -3.500 & 0.012 \\
\hline 10. & MACS-1505 & 19.30 & 6.496 & 0.020 & 39.18 & 8.646 & $0.386^{* *}$ \\
\hline 11. & AMS-100-39-1 & 20.61 & 3.408 & -0.031 & 35.39 & 6.107 & $0.204^{* *}$ \\
\hline 12. & MACS-1520 & 21.05 & 0.291 & -0.010 & 36.73 & -1.725 & $0.251^{* *}$ \\
\hline 13. & KDS-980 & 20.62 & -0.140 & -0.016 & 36.73 & 0.845 & $0.046^{*}$ \\
\hline 14. & MAUS-706 & 20.53 & 1.229 & -0.032 & 35.33 & 2.577 & -0.010 \\
\hline 15. & AMS-MB-5-19 & 20.94 & -0.291 & -0.012 & 35.97 & 0.078 & $0.050^{*}$ \\
\hline 16. & KDS-1045 & 19.86 & -3.712 & -0.027 & 37.15 & -3.527 & 0.008 \\
\hline 17. & MAUS-710 & 20.73 & 1.595 & -0.022 & 34.13 & 1.691 & -0.005 \\
\hline 18. & JS-335(C) & 20.84 & 1.622 & -0.027 & 34.77 & 3.322 & 0.005 \\
\hline 19. & JS-93-05(C) & 20.89 & 1.195 & -0.031 & 38.23 & 3.388 & -0.005 \\
\hline 20. & JS-97-52(C) & 20.18 & 3.367 & -0.030 & 37.32 & 8.674 & 0.015 \\
\hline 21. & MAUS-71(C) & 20.61 & 1.413 & -0.009 & 35.30 & 2.865 & 0.022 \\
\hline \multirow[t]{4}{*}{22.} & MAUS-158(C) & 20.65 & 2.055 & -0.025 & 37.05 & 2.742 & -0.004 \\
\hline & Grand mean & 20.42 & & & 36.64 & & \\
\hline & SEm \pm & 0.10 & & & 0.22 & & \\
\hline & SEb \pm & 2.01 & & & 5.53 & & \\
\hline
\end{tabular}

\subsection{Days to $50 \%$ flowering}

The data presented in (Table 3), indicated that out of 22 genotypes, 8 genotypes recorded high mean performance while 8 genotypes exhibited significant $\mathrm{S}^{2}$ di values. The genotypes, AMS-MB-5-19 exhibited higher mean, bi near to unity $(b i=1)$ and less deviation from regression line these genotype suitable under all environment. The genotypes, KS133 and JS-97-52 had high mean value than the general mean bi more than unity $(b>1)$ and non-significant $\left(S^{2} d i\right)$ indicating that there superior for favorable environment. The non linear 
component was significant which indicate the unpredictable performance over the environments. Joshi et al. (2005), Rao and Eswari (2006), Dhillon et al. (2009) showed both linear and non linear component significant for days to $50 \%$ flowering.

\subsection{Days to maturity}

The genotypes KDS-921 exhibited greater adaptability as their higher mean than the grand mean with bi around unity and non-significant $\left(s^{2}\right.$ di) value. The genotypes KDS-980 exhibited higher mean, bi less than one $(b<1)$ and less deviation from regression line these genotypes suitable under unfavorable environment. The genotype MACS-1520 showed low mean with bi around unity and non significant $S^{2}$ di suggesting their early maturity with wider adaptability. Non linear component was significant and of higher magnitude indicating its major contribution for expression of trait. Joshi et al. (2005), Rao and Eswari (2006), Dhillon et al. (2009) noticed reported that non linear component significant for days to maturity.

\subsection{Plant height}

Thirteen genotypes recorded higher mean plant height than grand mean out of which MACS-1543 had stable performance as it had bi near unity and non significant $S^{2}$ di indicating its suitability to varied environments. The Seven genotypes, AMS1002, MAUS-614, KS-133, MACS-1505, KDS-1045, KDS-980 and MAUS-71 recorded high mean with bi>1 and non-significant $\left(s^{2} \mathrm{di}\right)$ indicating that there superior for favorable environment. Rao and Eswari (2006), Dhillon et al., (2009), Tyagi et al. (2009) stressed that both linear and non linear component were significant for $\mathrm{G} \times \mathrm{E}$ interaction.

\subsection{No. of branches plant ${ }^{-1}$}

The genotypes, KDS-980 and MACS-1460 had higher mean than the grand mean bi near to unity $(\mathrm{bi}=1)$ and non-significant $\left(S^{2} \mathrm{di}\right)$ indicating that there superior for average response and stable under all environment. Among the genotypes, MACS1505, MAUS-740, KDS-1045, MACS-1520, MAUS-158, AMS100-39-1 and JS-335 had higher mean than the grand mean with bi was more than one (bi $>1)$ and non-significant $\left(s^{2} \mathrm{di}\right)$ indicating that there superior for favorable environment. The genotypes viz. MAUS-614, KDS-904 and JS-97-52 exhibited higher mean, bi less than one $(b<1)$ and less deviation from regression line these genotypes suitable under unfavorable environment. The non linear component was significant and of higher magnitude indicating its major contribution for expression of trait, Aremu et al. (2005), Rao and Eswari (2006), confirmed both linear and non linear component were significant for number of branches.

\subsection{No.of pods plant ${ }^{-1}$}

Ten genotypes exhibited high mean performance than general mean. Genotypes, AMS-100-39-1, KDS-1045 and MAUS-706 had higher mean than the grand mean bi near to unity $(b=1)$ and non-significant $\left(s^{2} \mathrm{di}\right.$ ) indicating that there superior for average response and stable under all environment. The genotypes viz. MACS-1460, KS-133, MACS-1543, AMS-MB-5-19 and MAUS-710 had higher mean than the grand mean bi more than unity $(b>1)$ and non-significant $\left(s^{2} d i\right)$ indicating that there superior for favorable environment. The significance of non linear component of G×E interaction indicated unpredictable genotypic performance over the environments, Mondal et al. (2005); Rao and Eswari (2006), Dhillon et al. (2009), Tyagi et al. (2009); Tyagi et al. (2011) reported significance of linear and non linear component for this trait.

\subsection{Number of seeds pod-1}

Genotypes viz. KDS-904, MAUS-740, MACS-1543, MAUS-614, MACS-1505, AMS-100-39-1, MACS-1520, KDS-980, AMSMB-5-19, JS-93-05 and MAUS-71 had higher mean than the grand mean bi more than unity $(b>1)$ and non-significant $\left(s^{2} \mathrm{di}\right)$ indicating that there superior for favorable environment. The genotypes, AMS-1002, AMS-1003, MAUS-706 and MAUS-158 had exhibited higher mean, bi less than one $(b<1)$ and less deviation from regression line these genotypes suitable under unfavorable environment. Mondal et al. (2005), Ramana et al. (2006), Dhillon et al. (2009); Tyagi et al. (2009) stressed both linear and non linear components significant for this trait.

\subsection{0 seed weight}

Eleven genotypes recorded higher mean than grand mean out of which two genotypes, MAUS-740, MAUS-614 showed high mean with bi around unity and non significant $S^{2}$ di values suggesting their adaptability to varied environment. Three genotypes, KDS-980, KDS-904, MAUS-706 had high mean with bi> 1 and non significant $S^{2}$ di values showing their suitability for rich environment. The genotypes, KS-133, JS-93-05, MAUS-71, MAUS-710 showed high mean with $\mathrm{bi}<1$ and non significant $S^{2}$ di values showing their suitability for poor environment. Significant non linear component of $\mathrm{G} \times \mathrm{E}$ contributed major portion of G x E. Rao and Eswari (2006), Dhillon et al., (2009), Tyagi et al. (2009) reported that both linear and non linear component were significant for this trait.

\subsection{Grain yield plant ${ }^{-1}(g)$}

It is revealed from table 15 that nine genotypes exhibited higher mean seed yield than grand mean out of which two genotypes MAUS-710, MAUS-740, were stable for varied environments as they recorded high mean values with bi near unity and non significant $S^{2}$ di values and genotypes, MACS-1543, MAUS-614, AMS-100-39-1, KDS-980, MAUS706 and AMS-MB-5-19 showed high mean with bi>1 and non significant $S^{2}$ di values suggesting their adaptability to favourable environment. The genotypes MACS-1460 showed high mean with $b i<1$ and non significant $S^{2}$ di values suggesting their adaptability to unfavourable environment. The pooled deviation and pooled error was significant suggesting its importance in expression of character. Mondal et al. (2005), and Ramana et al. (2006); Rao and Eswari (2006), Tyagi et al. (2009) reported both linear and non linear components showed significant for the traits. 


\subsection{Grain yield plot ${ }^{-1}(\mathrm{~kg})$}

The genotypes, MAUS-614, KDS-904, KDS-980 had higher mean than the grand mean bi near to unity $(b=1)$ and nonsignificant $\left(s^{2} d i\right)$ indicating that there superior for average response and stable under all environment. Genotypes MACS1460, MACS-1543, had higher mean than the grand mean bi more than unity $(b>1)$ and non-significant $\left(s^{2} d i\right)$ indicating that there superior for favorable environment. The pooled deviation and pooled error was significant suggesting its importance in expression of character. Mondal et al. (2005), Ramana et al. (2006); Tyagi et al. (2009) reported both linear and non linear components showed significant for grain yield per plot.

\subsection{Oil content (\%)}

The genotypes, JS-93-05, MAUS-706, showed high mean bi near unity and non significant $S^{2}$ di values indicating stability for this character over all environments. The nine genotypes viz. MACS-1460, KDS-904, MAUS-740, AMS-1002, AMS-10039-1, MAUS-710, JS-335 MAUS-71, MAUS-158, showed high mean with bi>1 and non-significant $\left(s^{2} d i\right)$ indicating that there superior for favorable environment. The genotypes viz. MACS-1520, KDS-980, AMS-MB-5-19 exhibited higher mean, bi less than one $(b<1)$ and less deviation from regression line these genotypes suitable under unfavorable environment. The significance of non linear component of $G \times E$ interaction indicated unpredictable genotypic performance over environments. Gurdeep Singh et al. (2003); Ramana et al. (2006) noted both linear and non linear component were significant for this trait.

\subsection{Protein content (\%)}

Twelve genotypes recorded higher mean than grand mean. The five genotypes, KDS-1045, JS-97-52, MAUS-740, JS-93-05, MAUS-158 exhibited high mean with bi more than one bi>1 and non significant $S^{2}$ di values showing their suitability for rich environment. The non linear component was significant for this character. Ramana et al. (2006) observed that both linear and non linear component were significant for this trait.

\section{Conclusion}

The promising genotypes can be released as new varieties after further testing or used as parents for generating new varieties with wide adaptability MAUS-740, MAUS-710 over environments or with specific adaptation (KDS-921, MACS1543, AMS-MB-5-19, KDS-1045, AMS-100-39-1, MACS-1460, JS-93-05, MAUS-706) to a particular environment for desirable attributes.

\section{Literature cited}

Alghamdi, S.S., 2004. Yield stability of some soybean genotype across diverse environments. Pakistan Journal of Biological Sciences 7(12), 2109-2114.

Aremu, C.O., Ojo, D.K., 2005. Genotype x environment interaction and selection for yield and related traits in soybean. Moor Journal of Agricultural Research 6(1-2), 81-86.

Arslanoghu, F., Aytac, S., 2010. Determination of stability and genotypexenvironment interactions of some agronomic properties in the different soybean cultivar. Bulgarian J. of Agricultural Sciences 16(2), 181-195.

Dhillon, S.K., Singh, G., Gill, B.S., Singh, G., 2009. Stability analysis for grain yield and its components in soybean. Crop Improvement 36(1), 35-58.

Eberhart, S.A., Russell, W., 1966. Stability parameters for comparing varieties. Crop Science 6, 30-40.

Finalay, K.W., Wilkinson, G.N., 1963. Analysis of adaptation in plant breeding programme. Australian Journal of Agricultural Research 14, 742-754.

Joshi, H.J., Vaghani, J.J., Mehta, D.R., 2005. Genotypexenvironment interaction and stability analysis in soybean. Advances in plant sciences 18(2), 515-518.

Mondal, S., Chettri, M., Nath, R., 2005. Stability of soybean lines for yield and yield attributing traits in hill zone of West Bengal. Indian Journal of Agricultural Science., 75(2), 106-108.

Ngalamu,T., Ashraf M., Meseka, S., 2013. Soybean (Glycine max L.) Genotype and Environment Interaction Effect on Yield and Other Related Traits, American Journal of Experimental Agriculture 3(4), 977-987.

Paroda, R.S., Hayes, J.D., 1971. An investigation of genotype $x$ environment interaction for rule of ear emergence in spring barley. Heredity 26, 157-175.

Plaisted, R.L., Peterson, L.C., 1959. A technique for evaluating the ability of selection to yield consistently in different locations and seasons. American Journal of Potato Research 36, 381-385.

Ramana, M.V., Satyanarayana, A., 2006. Stability analysis for quality characters in soybean. Legume Research 29(4), 274-277.

Rao, M.V.B., Eswari, K.B., 2006. Analysis of stability for some characters in soybean. International Journal of Agriculture Sciences 2(2), 559-561.

Sood, J.K., Jai, D., Daisy, A., Kumari, V., Sood, U.K., Singh, A., Singh, K., 2009. Genotypexenvironment interaction for yield and maturity in soybean. Crop improvement, 36(1), 59-63.

Gurdeep Singh, Dillon, S.K., Gill, B.S., Paheja, R.K., 2003. Stability analysis for oil yield and quality in soybean. Annals of Agricultural Research 24(2), 390-395.

Tyagi, S.D., Khan, M.H., 2009. Genotype x environment interaction and stability analysis for yield and its components in soybean. (Glycine max L. Merrill). Soybean Genetics newsletter, 36. 5-7.

Tyagi, S.D., Khan, M.H., Silva, J.A., 2011. Yield stability of some soybean genotypes across diverse environments. International Journal of Plant Breeding 5(1), 37-41. 